\title{
BET inhibitors block pancreatic stellate cell collagen I production and attenuate fibrosis in vivo
}

\author{
Krishan Kumar, ${ }^{1}$ Brian T. DeCant, ${ }^{1}$ Paul J. Grippo, ${ }^{2}$ Rosa F. Hwang, ${ }^{3}$ David J. Bentrem, ${ }^{4,5,6}$ \\ Kazumi Ebine, ${ }^{1}$ and Hidayatullah G. Munshi ${ }^{1,4,5,6}$ \\ 'Department of Medicine, Feinberg School of Medicine, Northwestern University, Chicago, Illinois, USA. ${ }^{2}$ Department \\ of Medicine, University of Illinois, Chicago, Illinois, USA. ${ }^{3}$ Department of Surgical Oncology, The University of Texas MD \\ Anderson Cancer Center, Houston, Texas, USA. ${ }^{4}$ Department of Surgery, Feinberg School of Medicine, Northwestern \\ University, Chicago, Illinois, USA. 5Jesse Brown VA Medical Center, Chicago, Illinois, USA. ${ }^{6}$ Robert H. Lurie Comprehensive \\ Cancer Center, Northwestern University, Chicago, Illinois, USA.
}

The fibrotic reaction, which can account for over $70 \%-80 \%$ of the tumor mass, is a characteristic feature of human pancreatic ductal adenocarcinoma (PDAC) tumors. It is associated with activation and proliferation of pancreatic stellate cells (PSCs), which are key regulators of collagen I production and fibrosis in vivo. In this report, we show that members of the bromodomain and extraterminal (BET) family of proteins are expressed in primary PSCs isolated from human PDAC tumors, with BRD4 positively regulating, and BRD2 and BRD3 negatively regulating, collagen I expression in primary cancer-associated PSCs. We show that the inhibitory effect of pan-BET inhibitors on collagen I expression in primary cancer-associated PSCs is through blocking of BRD4 function. Importantly, we show that FOSL1 is repressed by BRD4 in primary cancer-associated PSCs and negatively regulates collagen I expression. While BET inhibitors do not affect viability or induce PSC apoptosis or senescence, BET inhibitors induce primary cancer-associated PSCs to become quiescent. Finally, we show that BET inhibitors attenuate stellate cell activation, fibrosis, and collagen I production in the EL-Kras ${ }^{\mathrm{G} 12 \mathrm{D}}$ transgenic mouse model of pancreatic tumorigenesis. Our results demonstrate that $\mathrm{BET}$ inhibitors regulate fibrosis by modulating the activation and function of cancer-associated PSCs.

Conflict of interest: The authors have declared that no conflict of interest exists.

Submitted: April 12, 2016 Accepted: December 30, 2016 Published: February 9, 2017

Reference information: JCI Insight. 2017;2(3):e88032. doi:10.1172/ji.insight.88032.

\section{Introduction}

Pancreatic ductal adenocarcinoma (PDAC) is the most common form of pancreatic cancer, with a 5-year survival rate of approximately 7\% (1). The vast majority of PDAC patients present with locally advanced or metastatic disease, and the status quo treatments involve cytotoxic chemotherapy $(2,3)$. However, the success of current drug therapies is limited $(2,3)$, frequently only extending survival of PDAC patients by a few months at most $(4,5)$. As fibrotic reaction is a characteristic feature of the majority of PDAC tumors (6-8), there has been increasing interest in understanding the role and regulation of the stroma in PDAC progression and in mediating response to therapy. While ablating the stroma results in worse outcomes in mouse models of PDAC (9), remodeling and normalizing the stroma can improve survival in transgenic mouse models $(10,11)$.

Pancreatic stellate cells (PSCs) are the predominant fibroblasts present in the pancreas and the key regulators of fibrosis in vivo $(7,12)$. In the quiescent state, PSCs demonstrate cytoplasmic lipid droplets and produce minimal amounts of collagen I and other extracellular matrix (ECM) proteins (12). However, when activated during pancreatic injury or during cancer development, the PSCs lose their cytoplasmic lipid droplets and dramatically increase ECM production. They also acquire a myofibroblast-like phenotype and express $\alpha$-smooth muscle actin ( $\alpha$-SMA) (12). Significantly, a recent report showed that inhibitors targeting the bromodomain and extraterminal (BET) family proteins block activation and function of hepatic stellate cells (13).

The BET family of proteins, which includes BRD2, BRD3, BRD4, and the testis-specific BRDT, regulates transcription of genes involved in several human diseases (14-16). These proteins bind to the 
acetylation motifs present in histones and enable recruitment of transcription factors and other chromatin regulators during RNA transcription $(14,15)$. Selective inhibitors of BET proteins (e.g., JQ1 and I-BET151; refs. 14, 15) compete with the acetyl-binding pockets of BRD proteins and affect RNA transcription. The effects of these inhibitors in solid tumors have been shown to be through repression of a number of different oncogenes (16-18). For example, we previously showed that the BET inhibitors repress c-MYC and FOSL1 in pancreatic cancer cells to limit tumor growth $(19,20)$. While the role of BET proteins in pancreatic cancer cells has been studied extensively (19-22), the role of BET inhibitors in regulating PSC function is less well understood.

In this report, we show that BRD proteins are expressed in primary PSCs isolated from human PDAC tumors, with BRD4 positively regulating, and BRD2 and BRD3 negatively regulating, collagen I expression in primary cancer-associated PSCs. We show that the inhibitory effect of pan-BET inhibitors on collagen I expression in primary cancer-associated PSCs is through blocking of BRD4 function. Importantly, we show that FOSL1 is repressed by BRD4 in primary cancer-associated PSCs and negatively regulates collagen I expression. While BET inhibitors do not affect viability or induce PSC apoptosis or senescence, BET inhibitors induce primary cancer-associated PSCs to become quiescent. Finally, we show that BET inhibitors attenuate stellate cell activation, fibrosis, and collagen I production in the EL-Kras ${ }^{\mathrm{G} 12 \mathrm{D}}$ transgenic mouse model of pancreatic tumorigenesis. Our results demonstrate that BET inhibitors regulate fibrosis by modulating the activation and function of cancer-associated PSCs.

\section{Results}

BET inhibitors decrease collagen I production by primary PSCS isolated from human PDAC tumors. Compared with adjacent normal pancreatic tissue, PDAC tumors demonstrate increased fibrosis and increased numbers of activated myofibroblasts in the fibrotic microenvironment (6-8). As a recent report showed that the BET family protein BRD4 mediates the profibrotic response in activated liver stellate cells (13), we evaluated human PDAC tumors for BRD4 expression. BRD4 staining was seen in all nuclei, including spindle-shaped nuclei typical of PSCs present in the human PDAC tumors (Figure 1A). Costaining of BRD4 and $\alpha$-SMA showed that BRD4 was seen in nuclei of $\alpha-\mathrm{SMA}^{+}$cells (Figure 1A), suggesting that BRD4 protein may also regulate the function of PSCs. To understand the extent to which BET proteins regulated stellate cell collagen I production, we treated an immortalized human PSC line with the BET inhibitors JQ1 and I-BET151 $(14,15)$. The BET inhibitors decreased COL1A1 and COL1A2 mRNA expression and collagen I protein expression (Figure 1B). We also isolated primary stellate cells from resected human PDAC specimens using the outgrowth culture assay (23). Treatment of primary cancer-associated PSCs with BET inhibitors decreased collagen I mRNA and protein expression (Figure 1, C and D, and Supplemental Figure 1A; supplemental material available online with this article; https://doi.org/10.1172/jci.insight.88032DS1).

Knockdown of BRD4, but not BRD2 or BRD3, decreases collagen I production by primary PSCs. We next evaluated the relative contribution of the different $B E T$ proteins (BRD2, BRD3, and BRD4) in regulating collagen I production in primary cancer-associated PSCs and in the immortalized PSC line. Knocking down BRD2 increased collagen I expression in primary cancer-associated PSCs (Figure 2, A-D, and Supplemental Figure 1B) but decreased collagen I expression in the immortalized cell line (Figure 2, E and F). In contrast, BRD3 knockdown increased collagen I expression in both the primary cancer-associated PSCs and the immortalized PSC line (Figure 2 and Supplemental Figure 1B). Notably, and consistent with the effects of BET inhibitors (Figure 1), BRD4 knockdown decreased collagen I expression in both the primary cancer-associated PSCs and the immortalized stellate cell line (Figure 2 and Supplemental Figure 1B).

BRD4, but not BRD2 or BRD3, mediates JQ1 suppression of collagen I production by primary cancer-associated PSCs. While the BET inhibitors are equally effective in blocking the function of BRD2, BRD3, and BRD4 $(14,15)$, the BRD proteins, however, differentially regulate collagen I expression in primary cancer-associated PSCs (Figure 2). BRD2 and BRD3 proteins inhibit collagen I expression, but BRD4 enhances collagen I expression. Thus, we evaluated the relative contribution of BRD2, BRD3, and BRD4 in mediating the effects of pan-BET inhibitors (Figure 3). We coknocked down BRD2, BRD3, and BRD4 in different combinations and then evaluated the effects of JQ1 on collagen I expression. Coknockdown of BRD2 and BRD3 in primary cancer-associated PSCs increased collagen I expression that was inhibited by JQ1. In contrast, coknockdown of BRD4 decreased collagen I expression that was not further inhibited by JQ1. These results indicate that BRD4 positively regulates collagen I expression 
A
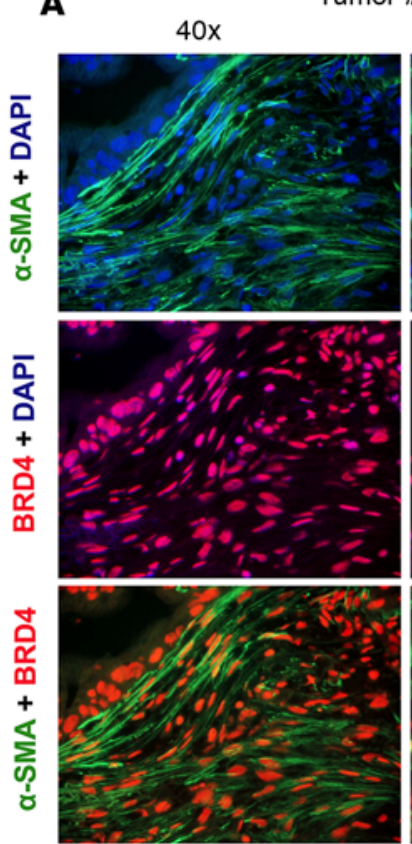

Tumor \#2

$40 x$

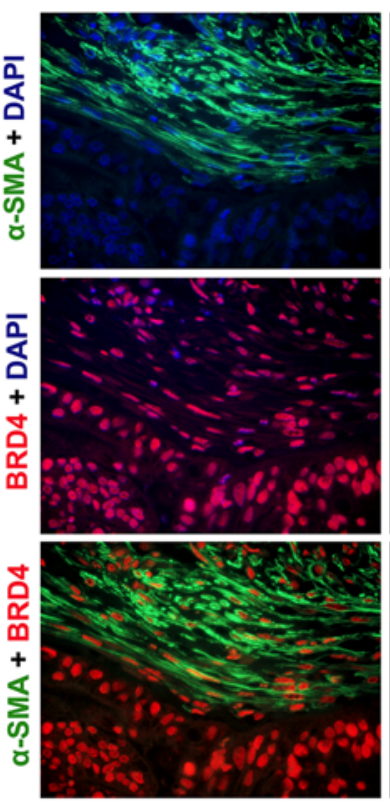

B
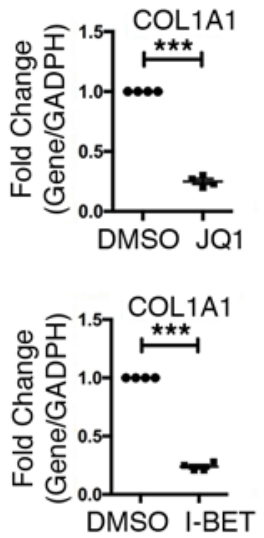

C
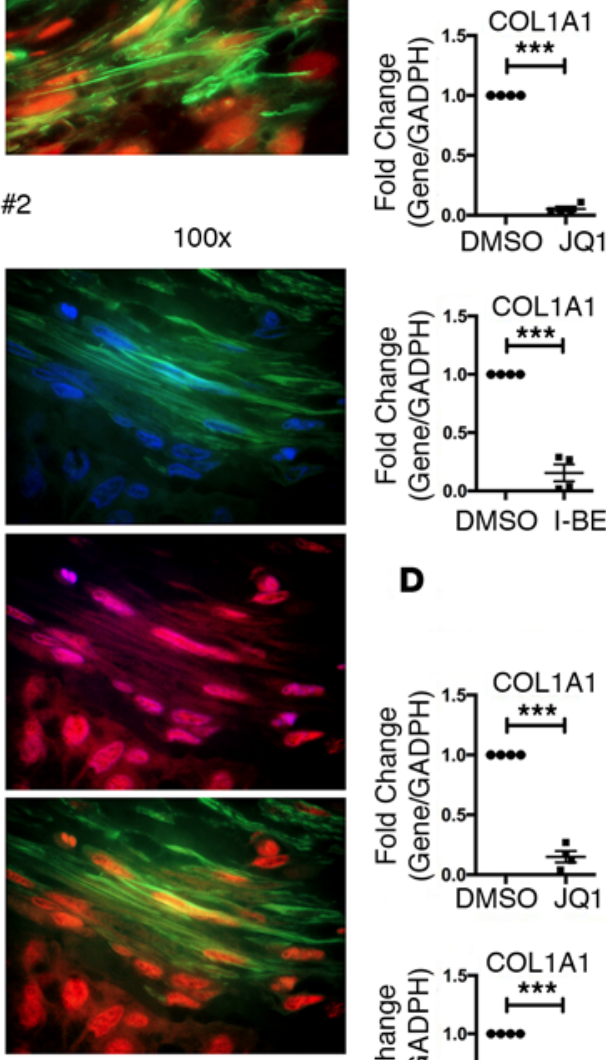
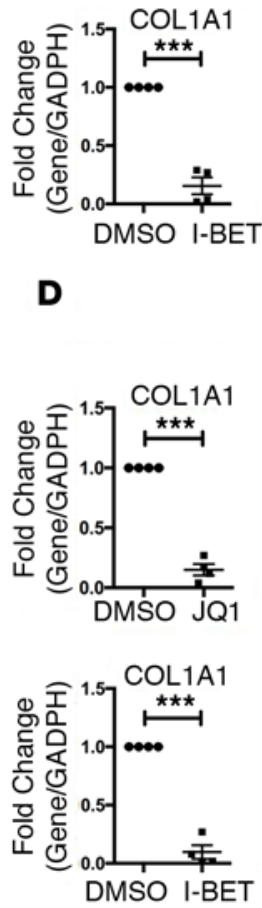

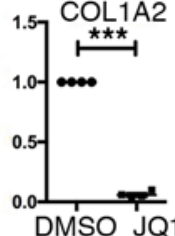

Stellate cell line

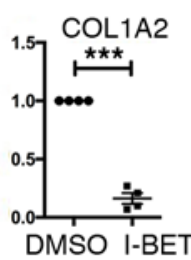

Primary PSCs \#1
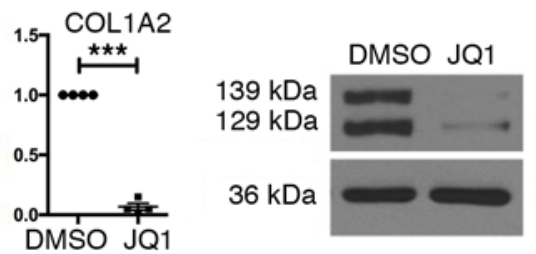

Collagen I

GAPDH

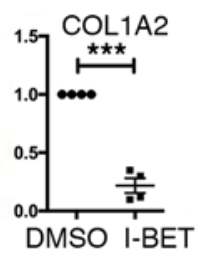

DMSO I-BET

$139 \mathrm{kDa}$ 129 kDa

$36 \mathrm{kDa}$

\section{DMSO I-BET}

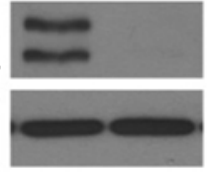

Collagen I

GAPDH

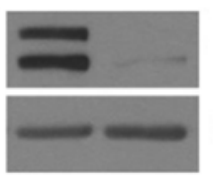

Collagen

GAPDH

Collagen

GAPDH

GAPDH

\section{Primary PSCs \#2}
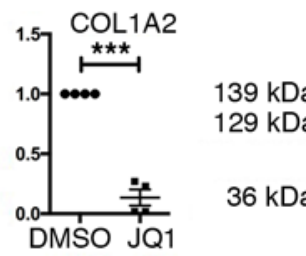

DMSO JQ1

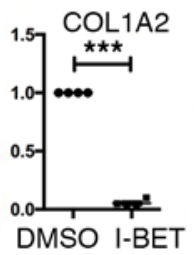

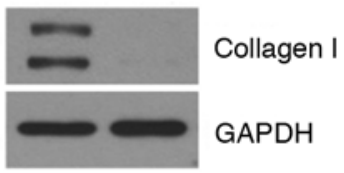

DMSO I-BET

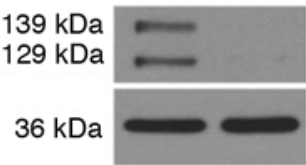

Collagen I

GAPDH

Figure 1. BET inhibitors decrease collagen I production by primary PSCs isolated from human PDAC tumors. (A) Human PDAC tumor sections were stained for $\alpha$-SMA and BRD4 by immunofluorescence, and nuclei were counterstained using DAPI. Original magnification, $\times 40$ (left); $\times 100$ (right). (B) An immortalized human pancreatic stellate cell line was treated with the BET inhibitors JQ1 (1 $\mu \mathrm{M})$ and I-BET151 (1 $\mu \mathrm{M})$, and the effect on COL1A1 and COL1A2 mRNA expression was determined by qRT-PCR $(n=4)$. ${ }^{* *} P<0.001$. Data were analyzed by 2 -tailed unpaired Student's $t$ test. The effect on collagen I protein expression was determined by Western blotting. These results are representative of $4(n=4)$ independent experiments. (C and $\mathbf{D})$ Early-passage cancer-associated primary PSCs, which were isolated from deidentified human PDAC specimens using the outgrowth assay, were treated with the BET inhibitors JQ1 $(1 \mu \mathrm{M})$ and I-BET151 $(1 \mu \mathrm{M})$. The effect on COL1A1 and COL1A2 mRNA expression was determined by qRT-PCR $(n=4)$. ${ }^{* * * P}<0.001$. Data were analyzed by 2-tailed unpaired Student's $t$ test. The effect on collagen I protein expression was determined by Western blotting. These results are representative of $4(n=4)$ independent experiments. BET, bromodomain and extraterminal; COL, collagen; PDAC, pancreatic ductal adenocarcinoma; PSCs, pancreatic stellate cells; $\alpha$-SMA, $\alpha$-smooth muscle actin. 
A
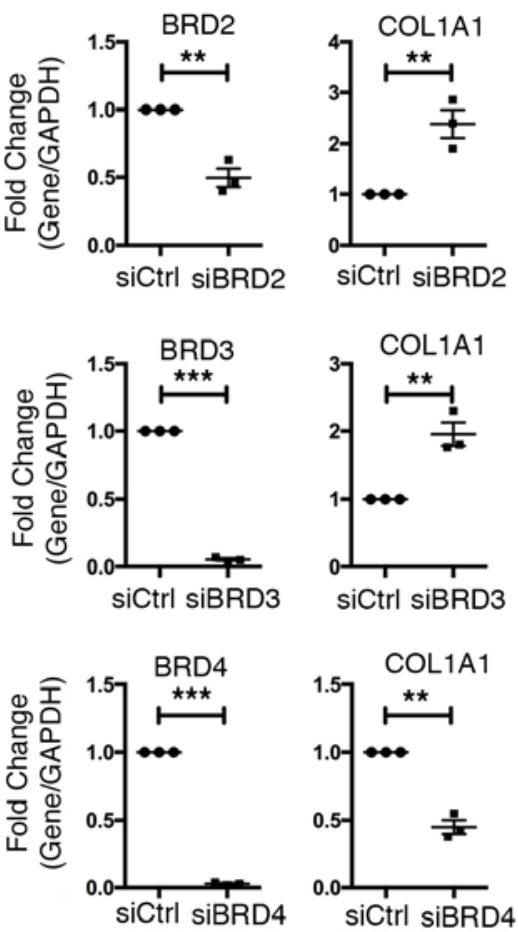

Primary PSCs \#2
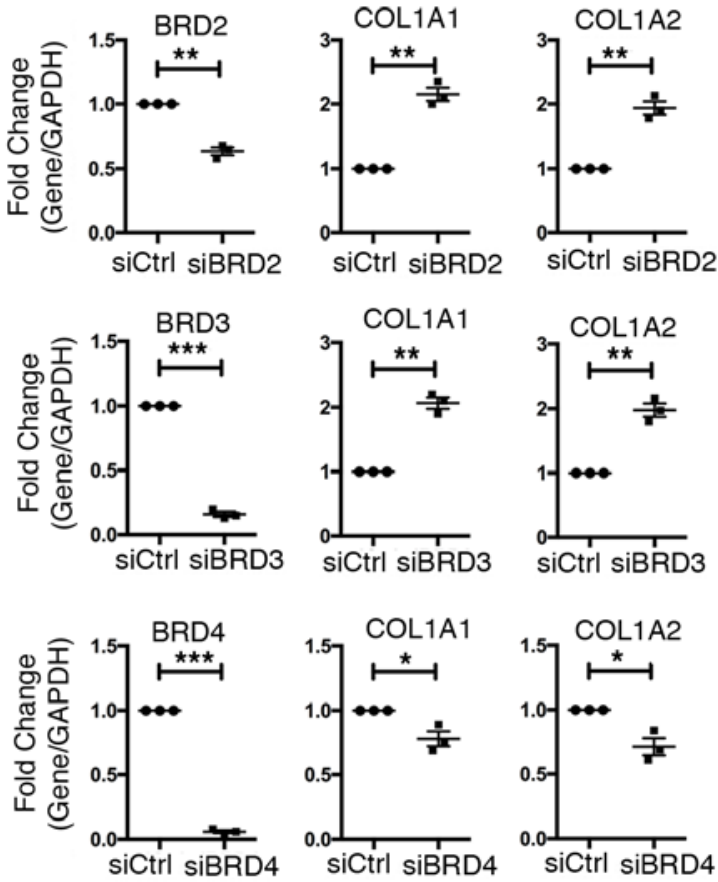

COL1A2
B

Primary PSCs\#1
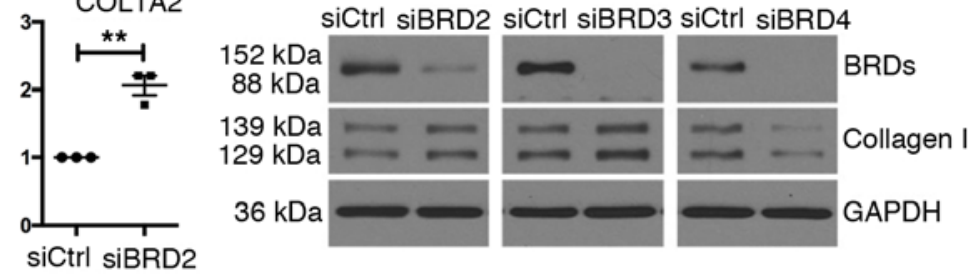

D

Primary PSCs \#2

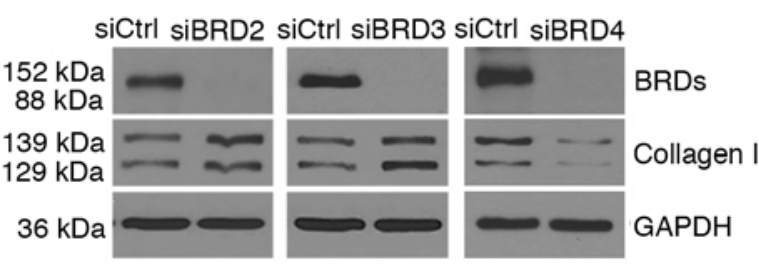

E

Stellate cell line
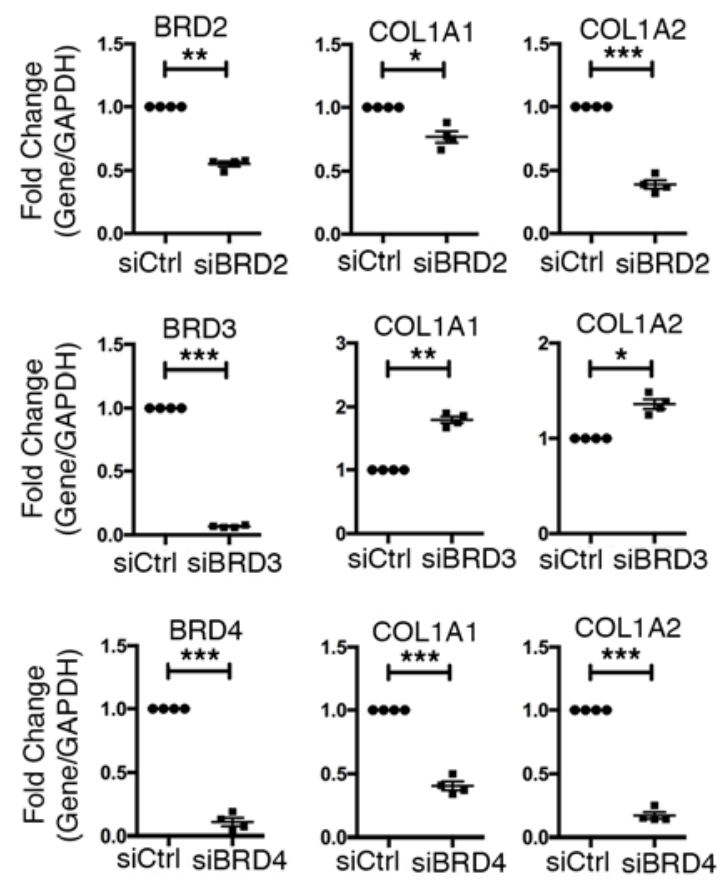

$\mathbf{F}$

Stellate cell line

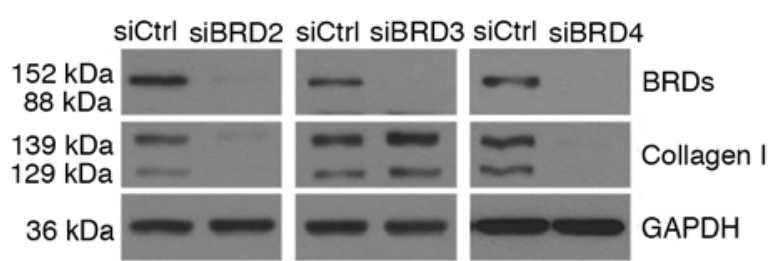

Figure 2. Knockdown of BRD4, but not BRD2 or BRD3, decreases collagen I production in primary cancer-associated PSCs and in an immortalized stellate cell line. Primary cancer-associated PSCs isolated from two different human PDAC tumors and an immortalized stellate cell line were transfected with control siRNA or with siRNAs against BRD2, BRD3, or BRD4. (A, C, and E) The effects on the individual BRD mRNAs and COL1A1 and COL1A2 mRNAs were determined by qRT-PCR ( $n=3$ for primary PSCs and $n=4$ for the immortalized stellate cell line). ${ }^{*} P<0.05,{ }^{* *} P<0.01$, ${ }^{* * *} P<0.001$. Data were analyzed by 2-tailed unpaired Student's $t$ test. (B, D, and $\mathbf{F}$ ) The effects on individual BRD proteins and collagen I protein expression were determined by Western blotting. These results are representative of $3(n=3)$ independent experiments for the primary PSCs and $4(n=4)$ independent experiments for the immortalized stellate cell line. COL, collagen; PDAC, pancreatic ductal adenocarcinoma; PSCs, pancreatic stellate cells. 

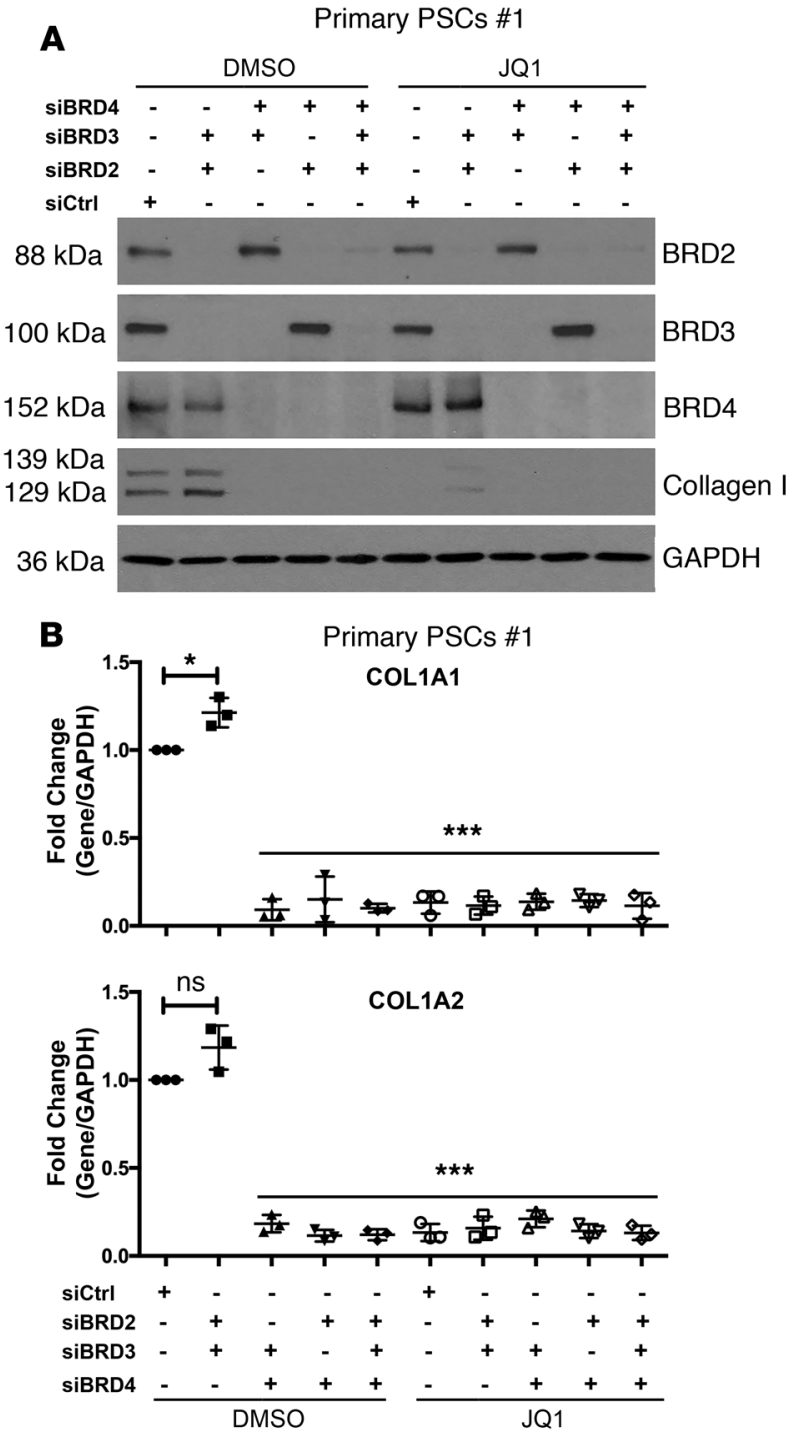
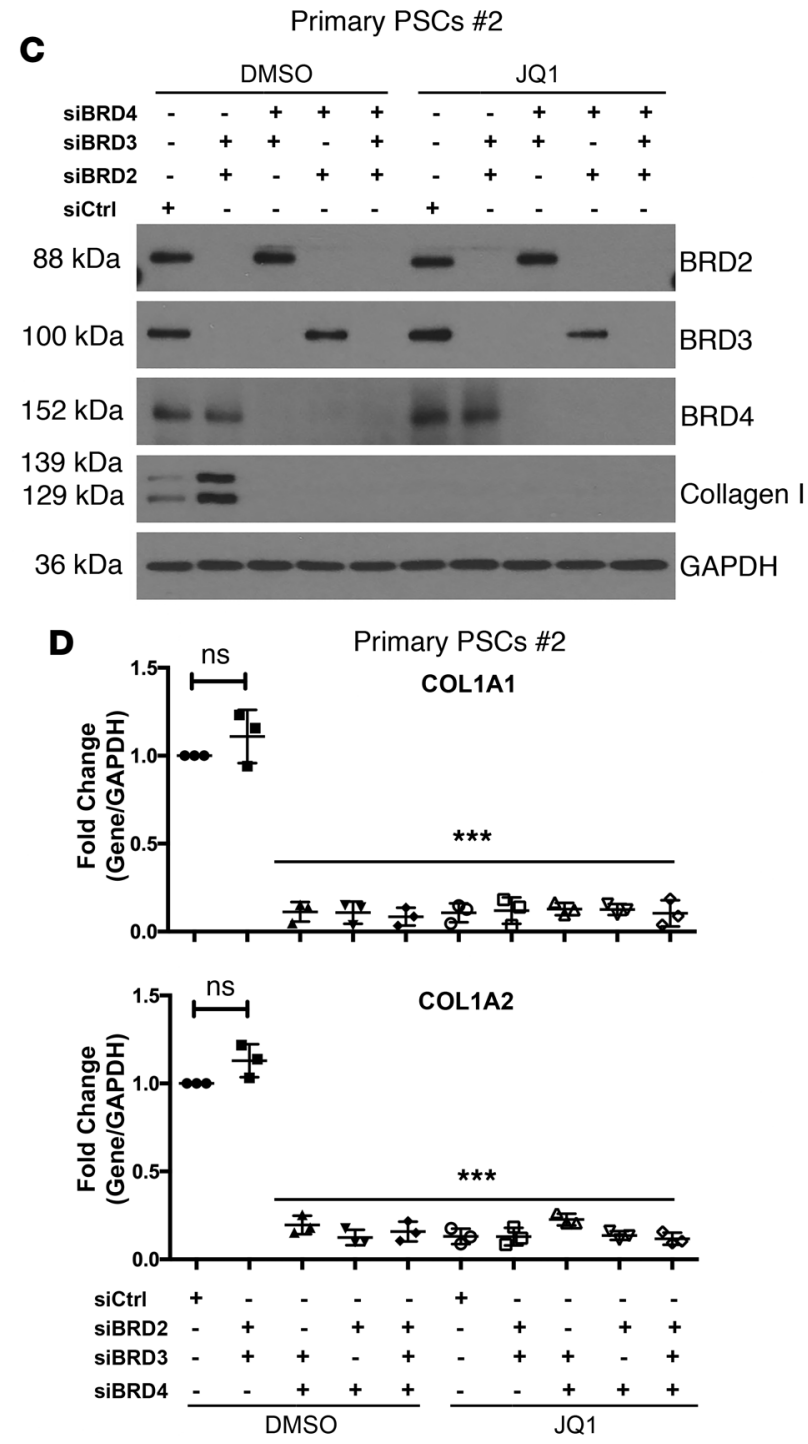

Figure 3. BRD4, but not BRD2 or BRD3, mediates JQ1 suppression of collagen I production by primary cancer-associated PSCs. Primary cancer-associated PSCs isolated from two different human PDAC tumors were transfected with control siRNA or with combination of siRNAs against BRD2, BRD3, and BRD4, and the cells were treated with DMSO or with the BET inhibitor JQ1 $(1 \mu \mathrm{M})$. (A and $\mathbf{C}$ ) The effects on individual BRD proteins and collagen I protein expression were determined by Western blotting. These results are representative of $3(n=3)$ independent experiments. (B and $\mathbf{D})$ The effects on COL1A1 and COL1A2 mRNA were examined by qRT-PCR $(n=3) .{ }^{*} P<0.05,{ }^{* * *} P<0.001$ (relative to siCtrl/DMSO samples). Data were analyzed by 2-tailed unpaired Student's $t$ test. BET, bromodomain and extraterminal; COL, collagen; PSCs, pancreatic stellate cells.

and that the inhibitory effect of pan-BET inhibitors on collagen I expression in primary cancer-associated PSCs is through blocking of BRD4 function.

FOSL1 is repressed by BRD4 in stellate cells and negatively regulates collagen I expression. BRD4 protein regulates expression of c-MYC, one of the main targets of BET inhibitors $(14,15)$, in a number of different cancers, including pancreatic cancer $(19,20)$. In addition, we have shown that BRD4 protein can regulate expression of FOSL1 in pancreatic cancer cells $(19,20)$. Thus, we evaluated to what extent BRD4 regulated c-MYC and FOSL1 in stellate cells and whether these proteins in turn regulated collagen I expression in stellate cells. BRD4 knockdown in the PSC line decreased c-MYC expression but increased FOSL1 expression (Figure 4A). c-MYC knockdown in the PSC line in turn decreased collagen I expression, but FOSL1 knockdown increased collagen I expression in the PSC line instead (Figure 4B). However, while BRD4 knockdown did not consistently increase c-MYC protein expression in the primary cancer-associated PSCs (Figure 4C and Supplemental Figure 2, A and C), knockdown of c-MYC in fact increased collagen I mRNA and protein expression in the primary PSCs (Figure 4D and Supplemental Figure 2, B and D). 
A

Stellate cell line siCtril siBRD4

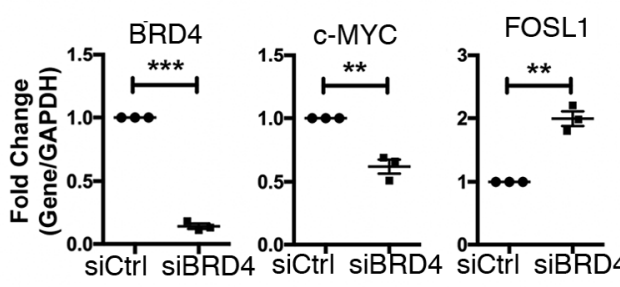

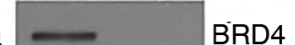

$60 \mathrm{kDa}$

$40 \mathrm{kDa}$

$36 \mathrm{kDa}$
D

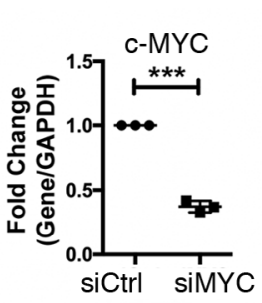

Primary PSCs \#1

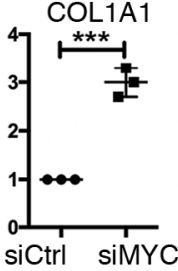

GAPDH

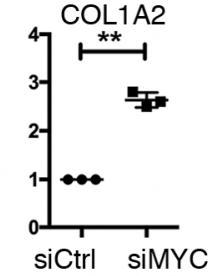

B
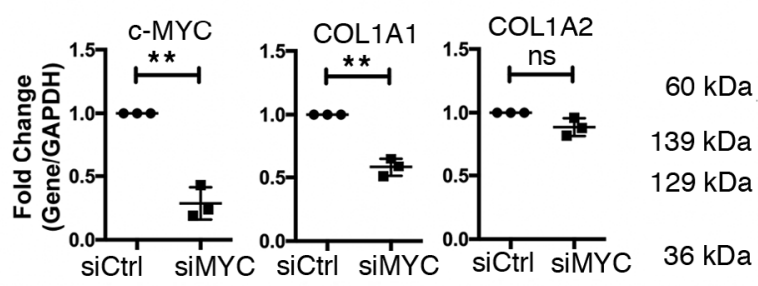

siCtrl siMYC

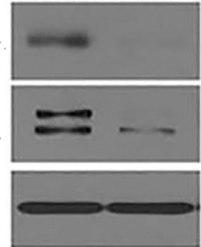
c-MYC

Collagen I

GAPDH
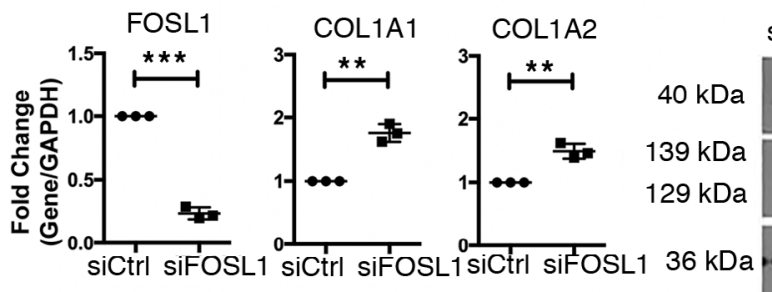

siCtrl siFOSL1

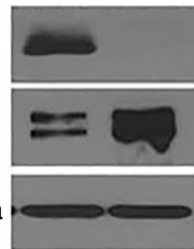

FOSL1

Collagen I

GAPDH
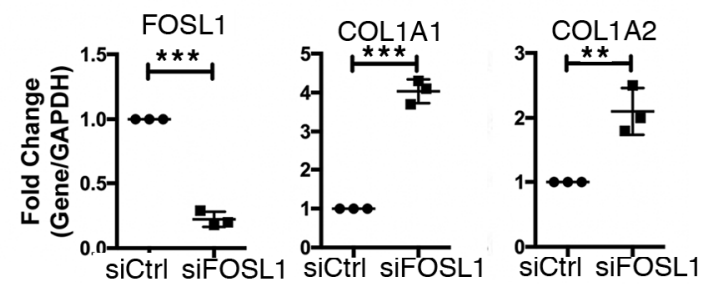

C

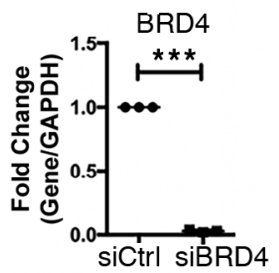

Primary PSCs \#1

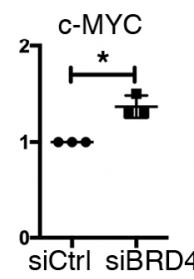

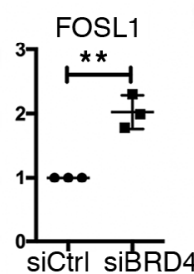

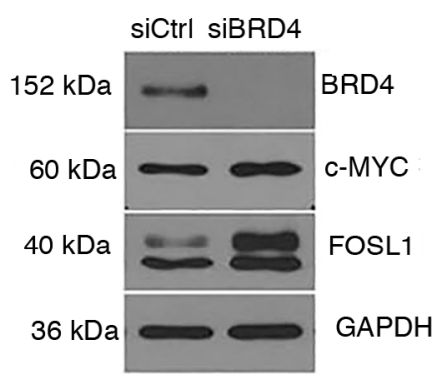

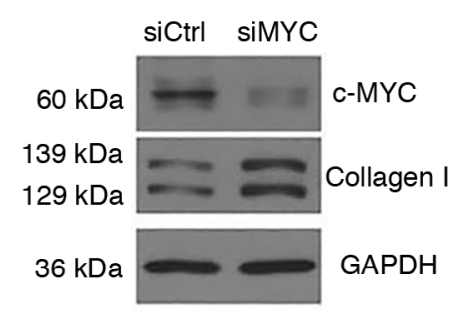

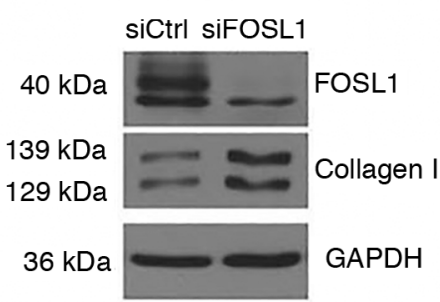

Figure 4. FOSL1 is repressed by BRD4 in stellate cells and negatively regulates collagen I expression. (A and C) BRD4 was knocked down in an immortalized pancreatic stellate cell line and in primary cancer-associated PSCs, and the effects on BRD4, c-MYC, and FOSL1 mRNA were determined by qRT-PCR $(n=3) .{ }^{*} P<0.05,{ }^{* *} P<0.01,{ }^{* *} P<0.001$. Data were analyzed by 2-tailed unpaired Student's $t$ test. The effects on BRD4, c-MYC, and FOSL1 protein expression were determined by Western blotting. These results are representative of $3(n=3)$ independent experiments. (B and D) c-MYC and FOSL1 were individually knocked down in an immortalized pancreatic stellate cell line and in primary cancer-associated PSCs, and the effects on c-MYC, FOSL1, COL1A1, and COL1A2 mRNA were determined by qRT-PCR $(n=3) .{ }^{* *} P<0.01,{ }^{* *} P<0.001$. Data were analyzed by 2-tailed unpaired Student's $t$ test. The effects on c-MYC, FOSL1, and collagen I protein expression were determined by Western blotting. These results are representative of $3(n=3)$ independent experiments. COL, collagen; PSCs, pancreatic stellate cells.

Similar to the findings in the immortalized stellate cell line, BRD4 knockdown in primary cancer-associated PSCs increased FOSL1 protein expression (Figure 4C and Supplemental Figure 2, A and C) and FOSL1 knockdown in turn increased collagen I expression in the primary cancer-associated PSCs (Figure 4D and Supplemental Figure 2, B and D).

$B R D 4$ regulates collagen I expression in primary cancer-associated PSCs following c-MYC and FOSL1 downregulation. We next evaluated the extent to which BRD4 mediates the effects of c-MYC and FOSL1 down- 
regulation on collagen I expression in the primary cancer-associated PSCs by cotransfecting c-MYC siRNA or FOSL1 siRNA with BRD4 siRNA. As shown previously (Figure 4), c-MYC knockdown increased collagen I expression. However, coknockdown of c-MYC and BRD4 decreased collagen I expression induced by c-MYC knockdown (Figure 5, A and B). Similarly, while FOSL1 knockdown increased collagen I expression, coknockdown of FOSL1 and BRD4 decreased collagen I expression induced by FOSL1 knockdown (Figure 5, C and D). Together, these results indicate that BRD4 regulates collagen I expression following c-MYC and FOSL1 downregulation in primary cancer-associated PSCs.

BET inhibitors do not affect viability or induce apoptosis or senescence of primary PSCS. To further understand the role of BET proteins in primary PSCs, we evaluated the effects of BET inhibitors on primary cancer-associated PSCs grown in 3D collagen (Figure 6A). The PSCs growing in 3D collagen and treated with DMSO demonstrated spindle-shaped elongated morphology. In contrast, the PSCs treated with JQ1 and I-BET151 displayed a rounded morphology (Figure 6A). Importantly, the primary cancer-associated PSCs treated with BET inhibitors did not undergo apoptosis (Figure 6B). Moreover, siRNA against BRD4, but not siRNAs against BRD2 or BRD3, blocked the spindle-shaped elongated morphology of PSCs in 3D collagen (Figure 6A). As with BET inhibitors, knockdown of BRD proteins in the primary cancer-associated PSCs did not induce apoptosis (Figure 6B). Furthermore, the primary cancer-associated PSCs treated with BET inhibitors were viable, as demonstrated by trypan blue exclusion assay (Figure 6C), and did not demonstrate evidence of increased senescence by $\beta$-galactosidase staining (Figure 6D). However, the morphological changes induced by BET inhibitors and BRD4 siRNA suggest that the BET inhibitors modulate stellate cell function.

BET inhibitors induce the primary cancer-associated PSCS to become quiescent. We next evaluated whether BET inhibitors affected PSC activation by evaluating the effects on $\alpha$-SMA expression. Treatment with BET inhibitors disrupted $\alpha$-SMA filament structure in the primary cancer-associated PSCs (Figure 7A) and decreased $\alpha$-SMA mRNA and protein levels (Figure 7B). We also evaluated whether treatment with BET inhibitors decreased cell cycle progression of the primary cancer-associated PSCs (24). Primary cancer-associated PSCs were treated with thymidine in order to synchronize their cell cycles. The cells were then washed with PBS to remove thymidine and grown in fresh media in the presence or absence of JQ1 with or without Click-iT EdU for 40 hours to label cells that are in S-phase. The cells were then costained with propidium iodide, and the percentage of S-phase cells was detected by flow cytometry. In the DMSO-treated PSCs, approximately $10 \%$ of cells were in the S-phase of the cell cycle, which was significantly reduced in the JQ1-treated PSCs (Figure 7C). As quiescent PSCs demonstrate increased lipid droplet (12), we also evaluated the effects of BET inhibitors on lipid droplet accumulation. Treatment with BET inhibitors induced accumulation of lipid droplets, as measured by Bodipy staining (Figure 7D). Together, these results suggest that BET inhibitors induce the primary cancer-associated PSCs to become quiescent.

BET inhibitors in vivo decrease fibrosis and collagen I expression in the EL-Kras ${ }^{G 12 D}$ mouse model of pancreatic tumorigenesis. Finally, we evaluated the effects of BET inhibitors in vivo on fibrosis in the EL-Kras ${ }^{\mathrm{G} 12 \mathrm{D}}$ transgenic mouse model, in which mutant Kras is expressed in the acinar cells of the pancreas (25-28). Two-month-old EL-Kras ${ }^{\mathrm{G} 12 \mathrm{D}}$ mice were treated with JQ1 for 4 weeks, and the pancreata were collected and analyzed for acinar-to-ductal metaplasia (ADM), fibrosis, stellate cell activation, and collagen I expression. JQ1 treatment decreased ADM, as determined by morphology, and decreased staining of the ductal marker CK19 (Figure 8, A and D). Trichrome staining demonstrated that mice treated with JQ1 showed decreased fibrosis that was also associated with decreased $\alpha$-SMA expression (Figure 8, B and D). Moreover, JQ1 treatment decreased collagen I expression in vivo (Figure 8, C and D). Together, these results demonstrate that the BET proteins regulate stellate cell activation and collagen I production in vitro and in vivo.

\section{Discussion}

Fibrosis plays an important role in pancreatic cancer progression and in response to therapy $(29,30)$. Previously, it was shown that attenuating fibrosis with short-term treatment with hedgehog inhibitors increased response to chemotherapy and prolonged survival of transgenic mice bearing PDAC tumors (31). However, depleting the stroma by ablating $\alpha$-SMA-expressing cells in the transgenic mouse models resulted in poorly differentiated tumors, increased metastases, and decreased survival (9). In contrast, normalizing the stroma, instead of depleting the stroma, with vitamin $\mathrm{D}$ treatment increased drug delivery to tumor cells and improved survival (10). Vitamin D induced the stellate cells to become quiescent, as reflected by reaccumulation of lipid droplets and decreased $\alpha$-SMA expression (10). In this report, we show that treatment with JQ1 decreases the stroma and decreases collagen I production by cancer-associated PSCs. It also induces 
A

Primary PSCs
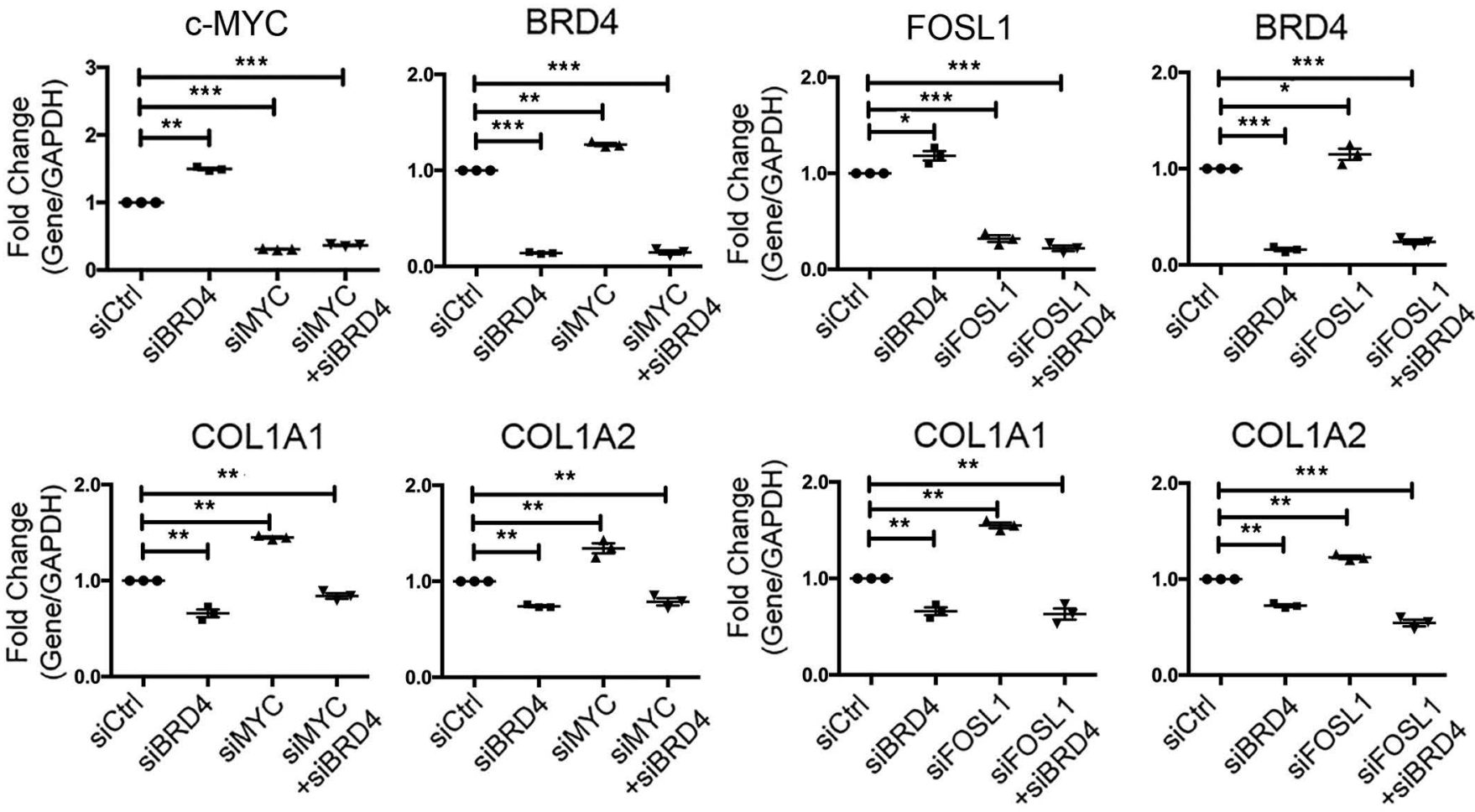

B

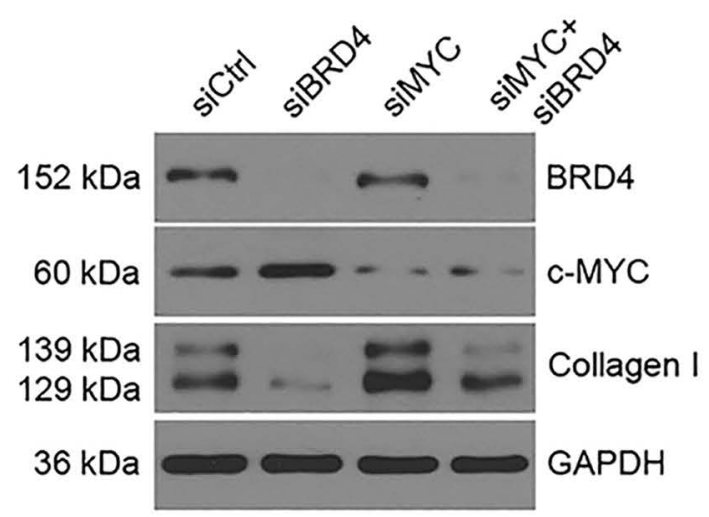

Primary PSCs

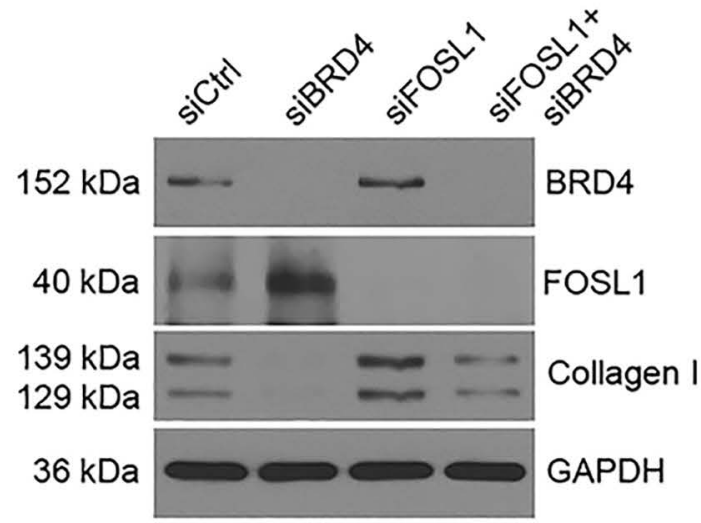

Figure 5. BRD4 regulates collagen I expression in primary cancer-associated PSCs following c-MYC and FOSL1 downregulation. (A and B) c-MYC and BRD4 were individually knocked down or coknocked down in primary cancer-associated PSCs, and the effects on c-MYC, BRD4, COL1A1, and COL1A2 mRNA were examined at the transcript level by qRT-PCR $(n=3) .{ }^{* *} P<0.01$, ${ }^{* *} P<0.001$. Data were analyzed by 2-tailed unpaired Student's $t$ test. The effect on c-MYC, BRD4, and collagen I protein expression was determined by Western blotting. These results are representative of $3(n=3)$ independent experiments. (C and D) FOSL1 and BRD4 were individually knocked down or coknocked down in primary cancer-associated PSCs, and the effects on FOSL1, BRD4, COL1A1, and COL1A2 mRNA were examined at the transcript level by qRT-PCR $(n=3)$. ${ }^{*} P<0.05,{ }^{*} P<0.01,{ }^{* * *} P<0.001$. Data were analyzed by 2 -tailed unpaired Student's $t$ test. The effect on FOSL1, BRD4, and collagen I protein expression was determined by Western blotting. These results are representative of $3(n=3)$ independent experiments. COL, collagen; PSCs, pancreatic stellate cells.

the primary cancer-associated PSCs to become quiescent, as shown by increased lipid droplet accumulation, decreased $\alpha$-SMA expression, and decreased cell cycle progression. Consistent with our findings, BET inhibitors were recently shown to cause hepatic stellate cells to become quiescent with increased lipid droplet accumulation and decreased collagen I production (13). Furthermore, our findings demonstrating that BET inhibitors do not induce primary cancer-associated PSCs to undergo apoptosis or senescence are in agreement with the lack of effect of BET inhibitors to induce apoptosis or senescence in hepatic stellate cells (13). 
A
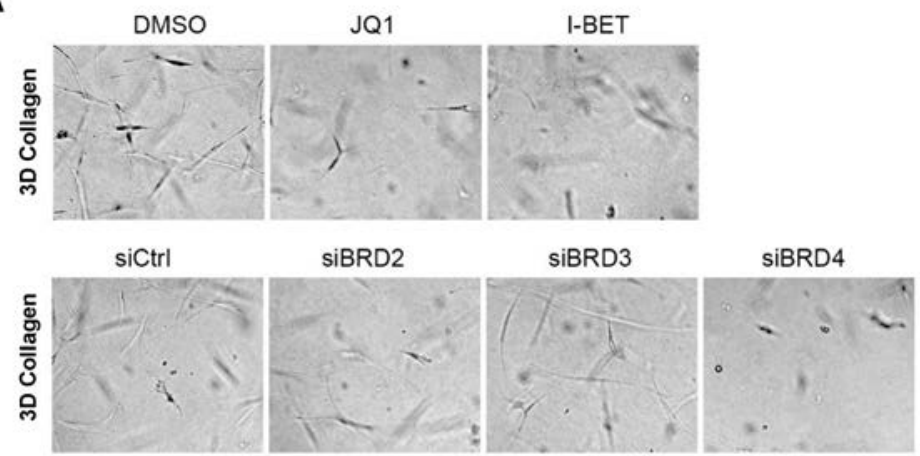

Primary PSCs

B

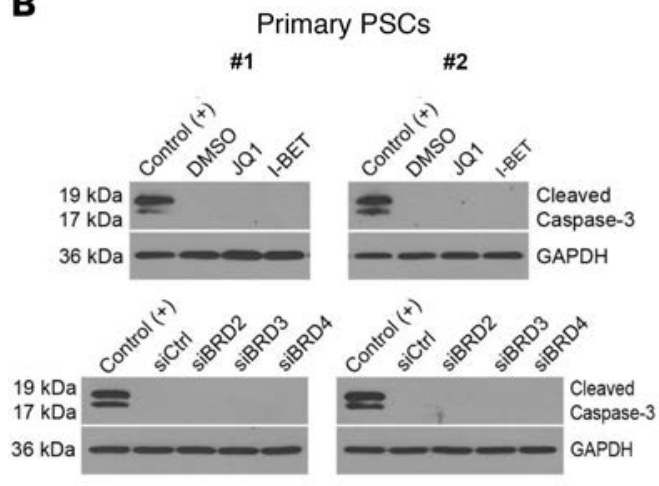

C

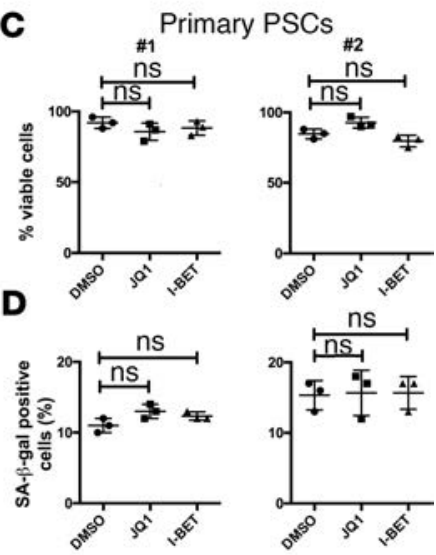

Figure 6. BET inhibitors do not affect viability or induce apoptosis or senescence of primary PSCs. (A) Primary cancer-associated PSCs were embedded in 3D collagen and treated with JQ1 $(1 \mu \mathrm{M})$ or I-BET151 (1 $\mu \mathrm{M})$ for 3 days. The effect on stellate cell morphology was assessed by phase-contrast microscopy. Primary cancer-associated PSCs were transfected with siRNAs against the individual BRDs for 48 hours and then grown in $3 \mathrm{D}$ collagen for an additional 3 days. Morphology of the stellate cells in $3 \mathrm{D}$ collagen was determined by phase-contrast microscopy (original magnification, $\times 50$ ). These results are representative of $3(n=3)$ independent experiments. (B) Primary cancer-associated PSCs were treated with the BET inhibitors JQ1 or I-BET151 (1 $\mu \mathrm{M})$ for 72 hours or transfected with the individual siRNAs for 96 hours. The effect on apoptosis was determined by Western blotting for cleaved caspase-3, using lysates from pancreatic cancer cells treated with gemcitabine as positive control. These results are representative of $3(n=3)$ independent experiments. (C and D) Primary cancer-associated PSCs were treated with JQ1 (1 $\mu \mathrm{M})$ or I-BET151 $(1 \mu \mathrm{M})$ for 3 days. Cell viability was assessed by trypan blue exclusion ( $n=3)$, and the effect on cellular senescence was measured by $\beta$-galactosidase staining $(n=3)$. Data were analyzed by 2-tailed unpaired Student's $t$ test. BET, bromodomain and extraterminal; PSCs, pancreatic stellate cells.

PSCs can also affect cancer cells by secreting growth factors and cytokines and depositing collagen I-rich ECM. We have previously shown that pancreatic cancer cells upregulate proteases, undergo epithelial-mesenchymal transition, and become more migratory and invasive on encountering collagen I (32, 33). Since BET inhibitors significantly decrease collagen I expression by primary cancer-associated PSCs, our results suggest that JQ1 may also block PDAC progression indirectly by decreasing collagen I-rich ECM deposition. Importantly, JQ1 can also directly limit growth of PDAC cells in vitro in patient-derived xenograft models and in transgenic mouse models $(19,21,22,34)$. Our findings that JQ1 decreases ADM development in the EL-Kras ${ }^{\mathrm{G} 12 \mathrm{D}}$ mouse model in vivo are in agreement with the report showing that JQ1 decreas-

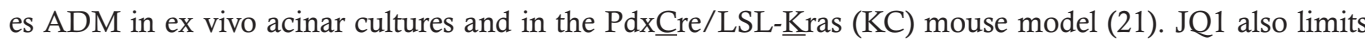
tumor development and attenuates $\alpha-\mathrm{SMA}^{+}$stroma in the $\mathrm{KC} / \mathrm{p} 53$ mouse model (21). Thus, BET inhibitors may block tumor progression directly by targeting cancer cells and indirectly by normalizing the stroma.

While many of the studies in cancer cells have evaluated the role of BRD4, the role of BRD2 and BRD3 has been less well studied. The few studies that have examined the relative roles of BRD proteins have shown that BRD proteins can have overlapping as well as distinct functions. For example, deletion of BRD2, similar to knockdown of BRD4, decreases erythroid gene activation (35). In contrast, deletion of BRD3 has no effect on erythroid gene activation (35). Importantly, while the phenotype of the BRD3 knockout mice has not been previously reported, both BRD2 and BRD4 are essential for normal development $(36,37)$. In this report, we characterized the relative role of the different BET proteins in regulation of collagen I expression in primary cancer-associated PSCs. We demonstrate a differential role of BRD2, $\mathrm{BRD} 3$, and BRD4 in the regulation of collagen I in stellate cells, with BRD4 positively regulating collagen I expression and BRD2 and BRD3 negatively regulating collagen I expression in primary cancer-associated PSCs. It is possible that BRD2 and BRD3 regulate expression of a protein that represses collagen I expression. Alternatively, loss of BRD2 or BRD3 results in increased BRD4 binding to the collagen I promoter, which results in an increased collagen I expression. Finally, even though BET inhibitors are equally effective in blocking the function of $\operatorname{BRD} 2$ and $\operatorname{BRD} 3(14,15)$, and, as a result, one would anticipate compensatory effects on collagen I expression, our results demonstrate that the effects of BET inhibitors on collagen I expression in primary cancer-associated PSCs are most closely modeled by BRD4 knockdown. 
A

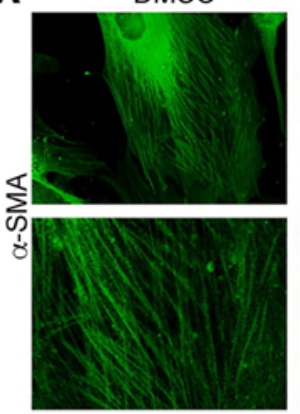

JQ1

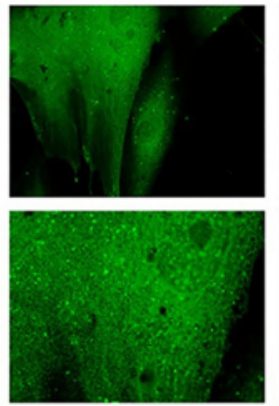

Primary PSCs
I-BET

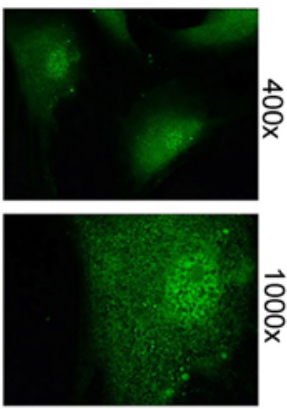

B $\alpha$-SMA
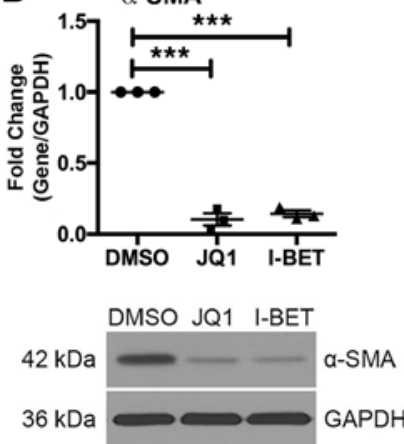

C

Primary PSCs

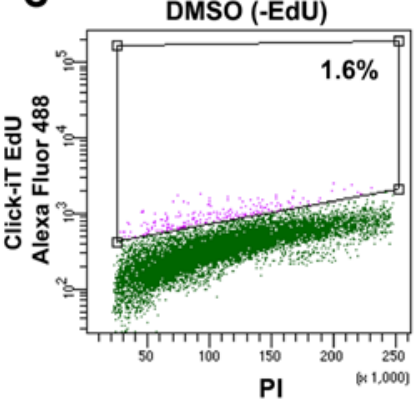

DMSO (+EdU)
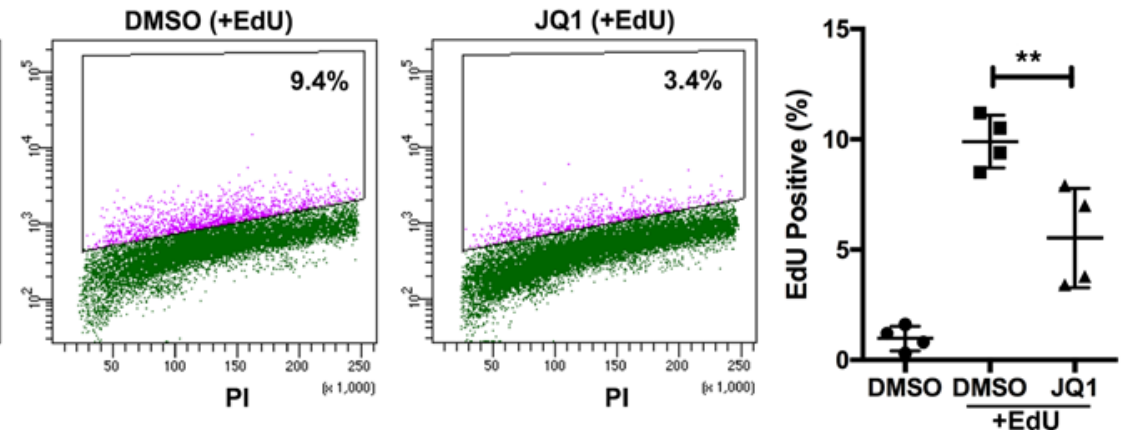

D

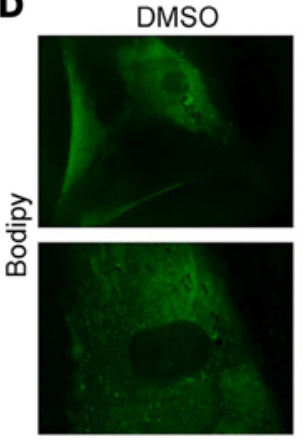

JQ1

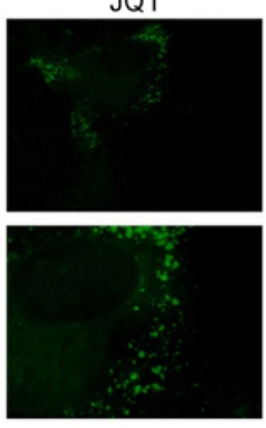

I-BET
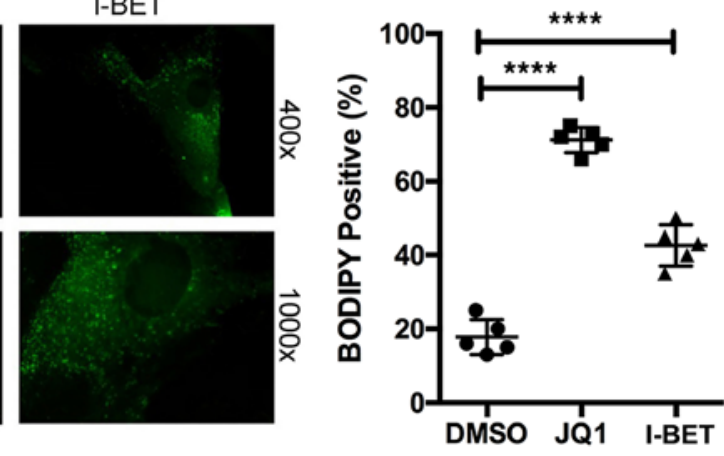

Primary PSCs

Figure 7. BET inhibitors induce primary cancer-associated PSCs to become quiescent. (A) Primary cancer-associated PSCs growing on glass coverslips were treated with the BET inhibitors JQ1 or I-BET151 $(1 \mu \mathrm{M})$ for 72 hours, and the effect on $\alpha$-SMA filaments was determined by immunofluorescence staining. Original magnification, $\times 400$ (top); $\times 1,000$ (bottom). These results are representative of $3(n=3)$ independent experiments. (B) Primary cancer-associated PSCs were treated with the BET inhibitors JQ1 $(1 \mu \mathrm{M})$ or I-BET151 $(1 \mu \mathrm{M})$ for 72 hours, and the effect on $\alpha$-SMA mRNA was determined by qRT-PCR $(n=3) .{ }^{* *} P<0.001$. Data were analyzed by 2 -tailed unpaired Student's $t$ test. The effect on $\alpha$-SMA protein expression was determined by Western blotting $(n=3)$. These results are representative of $3(n=3)$ independent experiments. (C) Primary cancer-associated PSCs were treated with thymidine ( $2 \mathrm{mM}$ ) for 24 hours to synchronize PSC cycle. The cells were then washed with PBS to remove thymidine and grown in fresh media in the presence of vehicle control (DMSO) or JQ1 $(1 \mu \mathrm{M})$ with or without Click-iT EdU $(2 \mu \mathrm{M})$ for additional 40 hours. The cells were then labeled using the ClickiT Alexa Fluor 488 Assay kit and costained with propidium iodide, and the percentage of S-phase cells was detected by flow cytometry $(n=4)$. ${ }^{* *} P<$ 0.01 . Data were analyzed by 2-tailed paired Student's $t$ test. (D) Primary cancer-associated PSCs growing on glass coverslips were treated with the BET inhibitors JQ1 $(1 \mu \mathrm{M})$ or I-BET151 $(1 \mu \mathrm{M})$ for 72 hours, and the effect on lipid droplet accumulation was determined using Bodipy $493 / 503$ fluorophore. Original magnification, $\times 400$ (top); $\times 1,000$ (bottom). The percentage of lipid-containing PSCs was quantified in 5 separate $(n=5)$ experiments. ${ }^{* * *} P<$ 0.0001 . Data were analyzed by 2-tailed unpaired Student's $t$ test. BET, bromodomain and extraterminal; COL, collagen; EdU, 5-ethynyl-2 deoxyuridine; PDAC, pancreatic ductal adenocarcinoma; PSCs, pancreatic stellate cells.

We demonstrate differences in collagen I regulation between the immortalized PSC line and the primary cancer-associated PSCs. In particular, we found that BRD2 knockdown increases collagen I expression in primary PSCs but decreases collagen I expression in the immortalized stellate cell line. We also found similar differences in collagen I regulation by c-MYC in the stellate cell line compared with the primary cancer-associated PSCs. Knockdown of c-MYC represses collagen I expression in the PSC line but increases collagen I expression in the primary cancer-associated PSCs. Importantly, it was previously shown that c-MYC in 
A
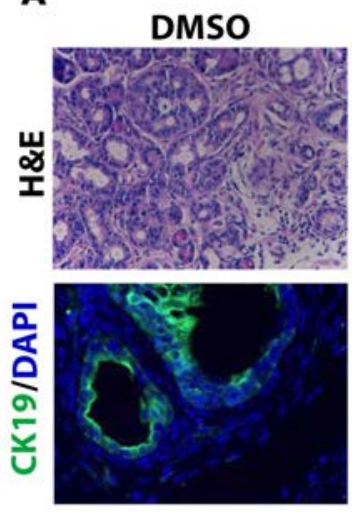

B
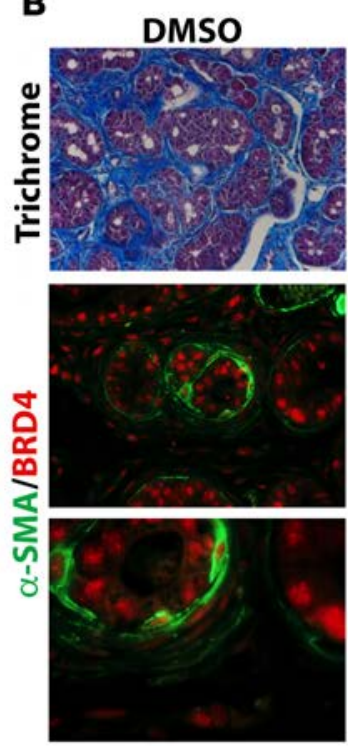

JQ1
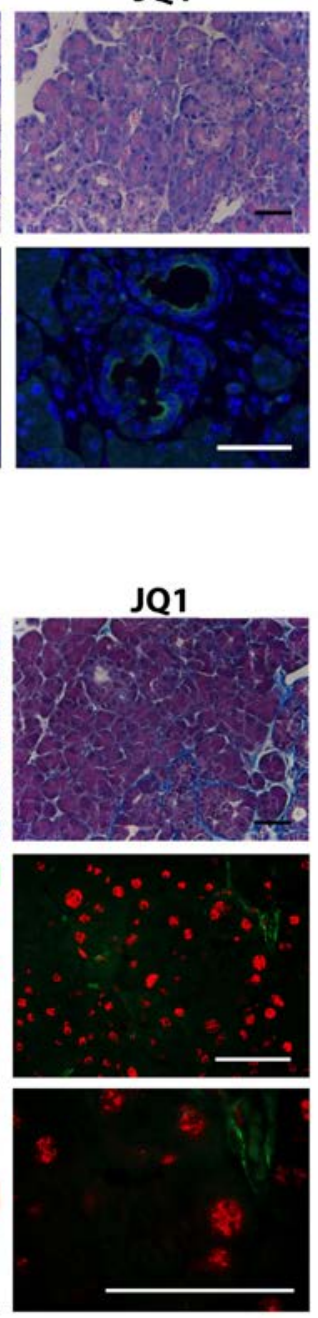

C
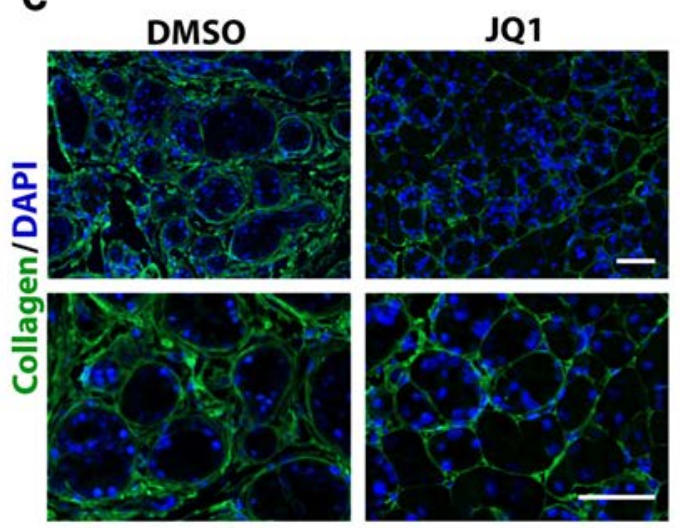

D
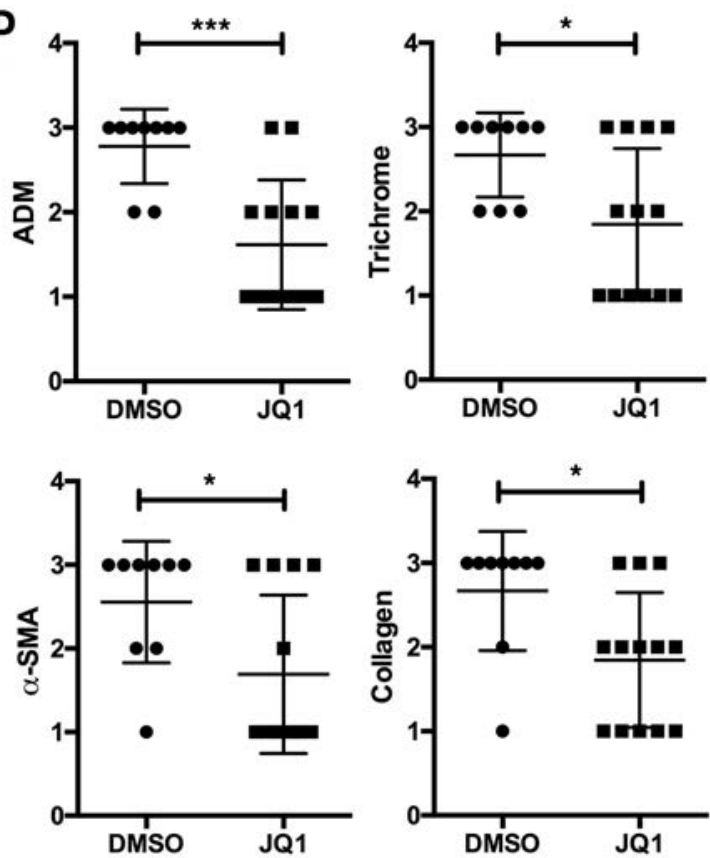

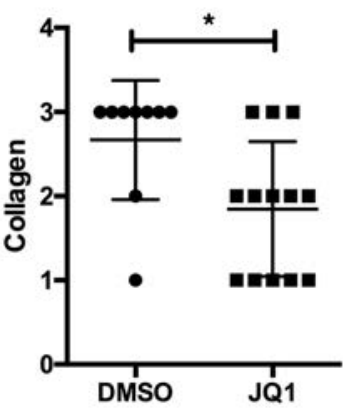

Figure 8. BET inhibitors decrease fibrosis and collagen production in the EL-Kras ${ }^{6120}$ mouse model of pancreatic tumorigenesis. Two-month-old EL-Kras ${ }^{\mathrm{G} 12 \mathrm{D}}$ mice, which express mutant Kras in the acinar cells, were i.p. injected with JQ1 (50 mg/ kg body weight, $n=13$ ) or vehicle control [DMSO/(2-hydroxypropyl)- $\beta$-cyclodextrin, $n=9$ ] daily for 5 days per week for 4 weeks. (A) The pancreatic tissue sections were stained with H\&E to observe phenotypic changes and additionally stained for the ductal marker CK19 together with DAPI to counterstain the nuclei. (B) The pancreatic sections were trichrome stained (blue = fibrosis) to assess for fibrosis. The pancreatic sections were also stained for $\alpha$-SMA to evaluate for stellate cell activation and costained for BRD4. (C) The sections were stained for collagen I expression, and the nuclei were costained with DAPI. (D) The extent of ADM and fibrosis, and the relative expression of $\alpha$-SMA and collagen I were quantified as described in Methods. ${ }^{*} P<0.05,{ }^{* *} P<0.001$. Data were analyzed by 2 -tailed unpaired Student's $t$ test. Scale bars: $50 \mu \mathrm{m}$. ADM, acinar-to-ductal metaplasia; $\alpha$-SMA, $\alpha$-smooth muscle actin; BET, bromodomain and extraterminal; CK19, cytokeratin 19.

mouse 3T3-L1 cells suppresses collagen I expression (38). While immortalized stellate cell lines may aid in our understanding of the role of stellate cells in cancer progression, immortalized stellate cell lines may not entirely reflect the role of BET proteins and the regulation of collagen I expression in stellate cells in vivo.

Importantly, we show that BRD4 negatively regulates FOSL1 both in the immortalized stellate cell line and in the primary PSCs. This is in contrast to previous findings in cancer cells derived from pancreatic and lung tumors, in which BRD4 was shown to positively regulate FOSL1 expression $(17,19)$. This difference in FOSL1 regulation may possibly be due to differences in genetic mutations or differences in signaling between cancer cells and cancer-associated PSCs. Of note, BRD proteins have been shown previously to function as transcriptional corepressors in different cell types. For example, BRD4 knockdown increases insulin mRNA 


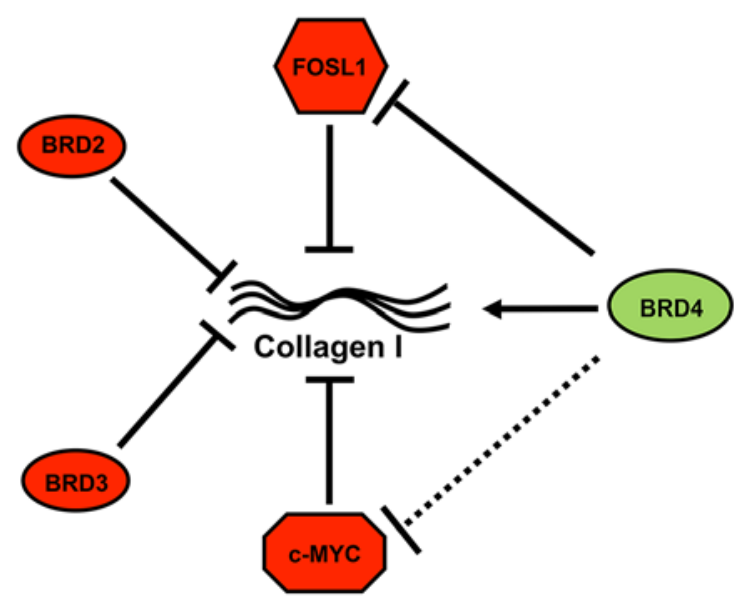

Figure 9. Model of regulation of collagen I in primary cancer-associated PSCs by BET family of proteins. BRD4 positively regulates collagen I expression in primary cancer-associated PSCs. In contrast, BRD2, BRD3, c-MYC, and FOSL1 negatively regulate collagen I expression in primary cancer-associated PSCs. In addition, BRD4 strongly represses FOSL1 in primary cancer-associated PSCs but has minimal effects on c-MYC expression.

and protein expression in pancreatic $\beta$ cells (39), while BRD2 knockdown increases peroxisome-proliferator-activated receptor- $\gamma$ in adipocytes (40). Importantly, we also show that FOSL1 is a negative regulator of collagen I in stellate cells. It has previously been shown that FOSL1 can also act as a transcriptional repres-

sor. FOSL1 inhibits B cell differentiation by binding to the BLIMP promoter and repressing its expression (41). In addition, FOSL1 blocks differentiation of an adipogenic cell line to adipocytes by binding to the $\mathrm{C} / \mathrm{EBP} \alpha$ promoter and repressing its expression (42). Our findings in the stellate cells are consistent with a previous report showing that FOSL1 represses collagen I expression in lung epithelial cells (43).

In summary, we show that BET inhibitors decrease fibrosis by modulating activation and collagen expression by cancer-associated PSCs (Figure 9). Given the importance of PSCs in regulating PDAC progression and response to therapy, BET inhibitors may have a role in the treatment of patients with pancreatic cancer and other diseases that are associated with a pronounced stromal reaction.

\section{Methods}

Chemicals/reagents. General tissue culture materials were obtained from VWR International. Antibodies against BRD2 (ab139690), BRD3 (ab50818), BRD4 (ab128874), and $\alpha$-SMA (ab5694) were obtained from Abcam. Antibodies against c-MYC (5605), FOSL1 (5281), and cleaved caspase-3 (9664) were purchased from Cell Signaling Technology. The collagen I antibody (1310-01) was from Southern Biotech; GAPDH antibody was from Millipore (MAB374); and the antibody against cytokeratin-19 (CK19; TROMA-III) was obtained from the University of Iowa. Secondary anti-mouse IgG (A4416) and anti-rabbit IgG (A6667) antibodies were purchased from Sigma-Aldrich. BET inhibitors JQ1 and I-BET151 were obtained from Tocris Bioscience. The siRNAs against BRD2, BRD3, BRD4, c-MYC, and FOSL1 were purchased from Life Technologies.

Cell culture. The human PSC line, which was immortalized with telomerase and SV40 large T antigen and characterized previously $(26,28,44)$, was obtained from Rosa F. Hwang (MD Anderson Cancer Center). Primary cancer-associated PSCs were generated from deidentified fresh human PDAC specimens using primary outgrowth cultures, which have been shown to be a reliable source of human PSCs (23). The primary cancer-associated PSCs were used within 5 to 6 passages from isolation.

Cell viability assay. Primary cancer-associated PSCs were treated with JQ1 (1 $\mu \mathrm{M})$ or I-BET151 $(1 \mu \mathrm{M})$ for 3 days, and the cell viability was determined using trypan blue exclusion test (45).

Cell senescence staining. Primary cancer-associated PSCs growing in 6-well plates were treated with JQ1 $(1 \mu \mathrm{M})$ or I-BET151 $(1 \mu \mathrm{M})$ for 3 days and then stained for senescence using a $\beta$-galactosidase staining kit (Cell Signaling, 9860s).

$S$-phase analysis. Primary cancer-associated PSCs were treated with thymidine (2 mM) for 24 hours to synchronize PSC cell cycle (46). The cells were then washed with PBS to remove thymidine and grown in fresh media in the presence of vehicle control (DMSO) or JQ1 $(1 \mu \mathrm{M})$ with or without ClickiT EdU $(2 \mu \mathrm{M})$ for additional 40 hours. The cells were then labeled using the Click-iT Alexa Fluor 488 Assay kit (C10420, Thermo Fisher Scientific) and costained with the Millipore propidium iodide kit (MCH100106). The percentage of cells in S-phase was then quantified by flow cytometry.

Transfection. The stellate cell line and the primary cancer-associated PSCs were transfected with siRNA against BRD2, BRD3, BRD4, c-MYC, FOSL1, or control siRNA (20 nM) using RNAimax (Invitrogen) according to the manufacturer's instructions $(20,47)$. 
qRT-PCR analysis. Quantitative gene expression was performed with gene-specific TaqMan probes, TaqMan Universal PCR Master Mix, and the 7500 Fast Real-time PCR System from Applied Biosystems. The data were then quantified with the comparative $C_{T}$ method for relative gene expression $(20,47)$.

Immunoblotting. Immunoblotting for BRD2, BRD3, BRD4, c-MYC, FOSL1, $\alpha$-SMA, $\alpha$-tubulin, and collagen I was done as described previously $(20,47)$.

Embedding and examination of cells in $3 D$ type I collagen gels. Collagen I mixture $(2 \mathrm{mg} / \mathrm{ml})$ was made by adding the appropriate volumes of sterile water, $10 \times \mathrm{DMEM}$, and $\mathrm{NaOH}$, and the mixture was kept on ice until needed $(20,47)$. Primary cancer-associated PSCs were then suspended in the collagen solution and allowed to gel at $37^{\circ} \mathrm{C}$. For morphologic examination of cells, PSCs growing in 3D collagen were examined using a Zeiss Axiovert $40 \mathrm{CFL}$ microscope, and pictures were taken with a Nikon Coolpix 4500 camera $(20,47)$.

Transgenic mice. EL-Kras ${ }^{\mathrm{G} 12 \mathrm{D}}$ mice (25-28), which express constitutively active mutant Kras in the pancreatic acinar cells, were obtained from Paul J. Grippo (University of Illinois). EL-Kras ${ }^{\mathrm{G} 12 \mathrm{D}}$ mice were i.p. injected with JQ1 $(50 \mathrm{mg} / \mathrm{kg})$ daily for 5 days per week for 4 weeks $(14,15)$ and processed for histochemistry and immunofluorescence studies.

Histochemistry. For the animal studies, the extent of ADM, as defined by vacuolization of the normal acini with formation of abnormal ducts along with evidence of fibrosis, was assessed, and a score of less than $25 \%(1+), 25 \%$ to $75 \%(2+)$, or more than $75 \%(3+)$ of the pancreas containing ADM was assigned to each mouse $(26,28)$. Mouse pancreatic samples were trichrome stained to assess for fibrosis and quantified as described previously $(26,28)$.

Immunohistochemistry and immunofluorescence. Human PDAC specimens were stained for $\alpha$-SMA and BRD4 by immunofluorescence together with DAPI to counterstain the nuclei. The pancreatic tissue specimens from EL-Kras ${ }^{\mathrm{G} 12 \mathrm{D}}$ mice were stained by immunofluorescence for CK19, collagen I, $\alpha$-SMA, and BRD4 and with DAPI to counterstain the nuclei. Antigen retrieval was carried out as previously described $(26,28)$. Expression of $\alpha$-SMA was graded, and a score of less than $25 \%(1+), 25 \%$ to $75 \%$ $(2+)$, or more than $75 \%(3+)$ of the pancreas was assigned to each mouse. Collagen I expression was graded based on low $(1+)$, medium $(2+)$, or high $(3+)$ collagen I staining in each mouse. In addition, primary PSCs growing on glass coverslips and treated with BET inhibitors were stained by immunofluorescence for lipid droplets using Bodipy stain. Briefly, the stellate cells were fixed in $10 \%$ formalin for 15 minutes and then stained with $1 \mu \mathrm{g} / \mathrm{ml}$ BODIPY 493/503 (Molecular Probes) for 1 hour at room temperature (10). The slides were then mounted and observed using a GFP filter, and the percentage of lipid-containing PSCs was quantified. Photographs for quantitative comparison were taken using a FeinOptic microscope and Jenoptik ProgRes C5 camera.

Statistics. The in vivo and in vitro results were compared using 2-tailed $t$ test analysis. Error bars represent standard error of the mean. All statistical analyses were done using GraphPad Instat. A $P$ value of less than 0.05 was considered significant.

Study approval. All animal work and procedures were approved by the Northwestern University Institutional Animal Care and Use Committee. In addition, all animal experiments were performed in accordance with relevant guidelines and regulations. For human studies, pancreatic tissue was obtained from patients with PDAC undergoing resection on a protocol approved by the Institutional Review Board of Northwestern University. Informed consent was obtained from patients prior to resection. The resected specimens, which were processed for histology and immunohistochemistry studies as well as for isolation of primary stellate cells, were deidentified.

\section{Author contributions}

$\mathrm{KK}, \mathrm{BTD}$, and KE performed the experiments, analyzed the data, and wrote and edited the manuscript. PJG provided EL-Kras ${ }^{G 12 D}$ mice and helped with the analysis of the mouse experiments. RFH provided the immortalized PSC line. DJB helped with procuring of the human PDAC specimens. HGM designed the studies, analyzed the data, and wrote and edited the manuscript.

\section{Acknowledgments}

This work was supported by grants (R01CA186885 and R01CA186885-S1 to HGM) from the National Cancer Institute, a Merit award (I01BX001363 to HGM) from the Department of Veterans Affairs, and an Acceleration Award from the Lurie Cancer Center. 
Address correspondence to: Krishan Kumar or Hidayatullah G. Munshi, Department of Medicine, Feinberg School of Medicine, Northwestern University, 303 E. Superior Avenue, Lurie 3-220 (K. Kumar) or Lurie 3-117 (H.G. Munshi), Chicago, Illinois 60611, USA. Phone: 312.503.0312; E-mail: krishan.kumar@northwestern. edu (K. Kumar). Phone: 312.503.2301; E-mail: h-munshi@northwestern.edu (H.G. Munshi).

1. Siegel RL, Miller KD, Jemal A. Cancer statistics, 2016. CA Cancer J Clin. 2016;66(1):7-30.

2. Vincent A, Herman J, Schulick R, Hruban RH, Goggins M. Pancreatic cancer. Lancet. 2011;378(9791):607-620.

3. Ryan DP, Hong TS, Bardeesy N. Pancreatic adenocarcinoma. N Engl J Med. 2014;371(11):1039-1049.

4. Conroy T, et al. FOLFIRINOX versus gemcitabine for metastatic pancreatic cancer. N Engl J Med. 2011;364(19):1817-1825.

5. Von Hoff DD, et al. Increased survival in pancreatic cancer with nab-paclitaxel plus gemcitabine. N Engl J Med. 2013;369(18):1691-1703.

6. Maitra A, Hruban RH. Pancreatic cancer. Annu Rev Pathol. 2008;3:157-188.

7. Shields MA, Dangi-Garimella S, Redig AJ, Munshi HG. Biochemical role of the collagen-rich tumour microenvironment in pancreatic cancer progression. Biochem J. 2012;441(2):541-552.

8. Whatcott CJ, et al. Desmoplasia in primary tumors and metastatic lesions of pancreatic cancer. Clin Cancer Res. 2015;21(15):3561-3568

9. Özdemir BC, et al. Depletion of carcinoma-associated fibroblasts and fibrosis induces immunosuppression and accelerates pancreas cancer with reduced survival. Cancer Cell. 2014;25(6):719-734.

10. Sherman $\mathrm{MH}$, et al. Vitamin D receptor-mediated stromal reprogramming suppresses pancreatitis and enhances pancreatic cancer therapy. Cell. 2014;159(1):80-93.

11. Provenzano PP, Cuevas C, Chang AE, Goel VK, Von Hoff DD, Hingorani SR. Enzymatic targeting of the stroma ablates physical barriers to treatment of pancreatic ductal adenocarcinoma. Cancer Cell. 2012;21(3):418-429.

12. Apte MV, Wilson JS, Lugea A, Pandol SJ. A starring role for stellate cells in the pancreatic cancer microenvironment. Gastroenterology. 2013;144(6):1210-1219.

13. Ding N, et al. BRD4 is a novel therapeutic target for liver fibrosis. Proc Natl Acad Sci U S A. 2015;112(51):15713-15718

14. Delmore JE, et al. BET bromodomain inhibition as a therapeutic strategy to target c-Myc. Cell. 2011;146(6):904-917.

15. Filippakopoulos P, et al. Selective inhibition of BET bromodomains. Nature. 2010;468(7327):1067-1073.

16. Sahai V, Redig AJ, Collier KA, Eckerdt FD, Munshi HG. Targeting bet bromodomain proteins in solid tumors. Oncotarget. 2016;7(33):53997-54009.

17. Lockwood WW, Zejnullahu K, Bradner JE, Varmus H. Sensitivity of human lung adenocarcinoma cell lines to targeted inhibition of BET epigenetic signaling proteins. Proc Natl Acad Sci USA. 2012;109(47):19408-19413.

18. Shu S, et al. Response and resistance to BET bromodomain inhibitors in triple-negative breast cancer. Nature. 2016;529(7586):413-417.

19. Sahai V, et al. BET bromodomain inhibitors block growth of pancreatic cancer cells in three-dimensional collagen. Mol Cancer Ther. 2014;13(7):1907-1917.

20. Kumar K, et al. GLI2-dependent c-MYC upregulation mediates resistance of pancreatic cancer cells to the BET bromodomain inhibitor JQ1. Sci Rep. 2015;5:9489.

21. Mazur PK, et al. Combined inhibition of BET family proteins and histone deacetylases as a potential epigenetics-based therapy for pancreatic ductal adenocarcinoma. Nat Med. 2015;21(10):1163-1171.

22. Roy N, et al. Brg1 promotes both tumor-suppressive and oncogenic activities at distinct stages of pancreatic cancer formation. Genes Dev. 2015;29(6):658-671.

23. Han S, et al. Primary outgrowth cultures are a reliable source of human pancreatic stellate cells. Lab Invest. 2015;95(11):1331-1340.

24. Froeling FE, et al. Retinoic acid-induced pancreatic stellate cell quiescence reduces paracrine Wnt- $\beta$-catenin signaling to slow tumor progression. Gastroenterology. 2011;141(4):1486-97, 1497.e1.

25. Grippo PJ, Nowlin PS, Demeure MJ, Longnecker DS, Sandgren EP. Preinvasive pancreatic neoplasia of ductal phenotype induced by acinar cell targeting of mutant Kras in transgenic mice. Cancer Res. 2003;63(9):2016-2019.

26. Shields MA, et al. Snail cooperates with KrasG12D to promote pancreatic fibrosis. Mol Cancer Res. 2013;11(9):1078-1087.

27. Knab LM, et al. Snail cooperates with Kras G12D in vivo to increase stem cell factor and enhance mast cell infiltration. Mol Cancer Res. 2014;12(10):1440-1448.

28. Krantz SB, et al. MT1-MMP cooperates with $\operatorname{Kras}(\mathrm{G} 12 \mathrm{D})$ to promote pancreatic fibrosis through increased TGF- $\beta$ signaling. Mol Cancer Res. 2011;9(10):1294-1304.

29. Gore J, Korc M. Pancreatic cancer stroma: friend or foe? Cancer Cell. 2014;25(6):711-712.

30. Rhim AD, et al. Stromal elements act to restrain, rather than support, pancreatic ductal adenocarcinoma. Cancer Cell. 2014;25(6):735-747.

31. Olive KP, et al. Inhibition of Hedgehog signaling enhances delivery of chemotherapy in a mouse model of pancreatic cancer. Science. 2009;324(5933):1457-1461.

32. Ottaviano AJ, Sun L, Ananthanarayanan V, Munshi HG. Extracellular matrix-mediated membrane-type 1 matrix metalloproteinase expression in pancreatic ductal cells is regulated by transforming growth factor-beta1. Cancer Res. 2006;66(14):7032-7040

33. Shields MA, Dangi-Garimella S, Krantz SB, Bentrem DJ, Munshi HG. Pancreatic cancer cells respond to type I collagen by inducing snail expression to promote membrane type 1 matrix metalloproteinase-dependent collagen invasion. J Biol Chem. 2011;286(12):10495-10504.

34. Garcia PL, et al. The BET bromodomain inhibitor JQ1 suppresses growth of pancreatic ductal adenocarcinoma in patient-derived xenograft models. Oncogene. 2016;35(7):833-845. 
35. Stonestrom AJ, et al. Functions of BET proteins in erythroid gene expression. Blood. 2015;125(18):2825-2834.

36. Houzelstein D, Bullock SL, Lynch DE, Grigorieva EF, Wilson VA, Beddington RS. Growth and early postimplantation defects in mice deficient for the bromodomain-containing protein Brd4. Mol Cell Biol. 2002;22(11):3794-3802.

37. Shang E, Wang X, Wen D, Greenberg DA, Wolgemuth DJ. Double bromodomain-containing gene Brd2 is essential for embryonic development in mouse. Dev Dyn. 2009;238(4):908-917.

38. Yang BS, Geddes TJ, Pogulis RJ, de Crombrugghe B, Freytag SO. Transcriptional suppression of cellular gene expression by c-Myc. Mol Cell Biol. 1991;11(4):2291-2295.

39. Deeney JT, Belkina AC, Shirihai OS, Corkey BE, Denis GV. BET Bromodomain proteins Brd2, Brd3 and Brd4 selectively regulate metabolic pathways in the pancreatic $\beta$-cell. PLoS One. 2016;11(3):e0151329.

40. Wang F, Liu H, Blanton WP, Belkina A, Lebrasseur NK, Denis GV. Brd2 disruption in mice causes severe obesity without Type 2 diabetes. Biochem J. 2009;425(1):71-83

41. Grötsch B, et al. The AP-1 transcription factor Fra1 inhibits follicular B cell differentiation into plasma cells. J Exp Med. 2014;211(11):2199-2212.

42. Luther J, et al. Elevated Fra-1 expression causes severe lipodystrophy. J Cell Sci. 2011;124(Pt 9):1465-1476.

43. Rajasekaran S, Vaz M, Reddy SP. Fra-1/AP-1 transcription factor negatively regulates pulmonary fibrosis in vivo. PLoS ONE. 2012;7(7):e41611.

44. Hwang RF, et al. Cancer-associated stromal fibroblasts promote pancreatic tumor progression. Cancer Res. 2008;68(3):918-926.

45. Strober W. Trypan blue exclusion test of cell viability. Curr Protoc Immunol. 2001;Appendix 3:Appendix 3B.

46. Harper JV. Synchronization of cell populations in G1/S and G2/M phases of the cell cycle. Methods Mol Biol. 2005;296:157-166.

47. Kumar K, et al. Differential regulation of ZEB1 and EMT by MAPK-interacting protein kinases (MNK) and eIF4E in pancreatic cancer. Mol Cancer Res. 2016;14(2):216-227. 